\title{
Computational analysis of an infinite magneto-thermoelastic solid periodically dispersed with varying heat flow based on non-local Moore-Gibson-Thompson approach
}

\begin{abstract}
In this investigation, a computational analysis is conducted to study a magneto-thermoelastic problem for an isotropic perfectly conducting half-space medium. The medium is subjected to a periodic heat flow in the presence of a continuous longitude magnetic field. Based on Moore-Gibson-Thompson equation, a new generalized model has been investigated to address the considered problem. The introduced model can be formulated by combining the Green-Naghdi Type III and Lord-Shulman models. Eringen's non-local theory has also been applied to demonstrate the effect of thermoelastic materials which depends on small scale. Some special cases as well as previous thermoelasticity models are deduced from the presented approach. In the domain of the Laplace transform, the system of equations is expressed and the problem is solved using state space method. The converted physical expressions are numerically reversed by Zakian's computational algorithm. The analysis indicates the significant influence on field variables of non-local modulus and magnetic field with larger values. Moreover, with the established literature, the numerical results are satisfactorily examined.
\end{abstract}

Communicated by Andreas Öchsner.

A. E. Abouelregal

Department of Mathematics, College of Science and Arts, Jouf University, Al-Qurayyat, Saudi Arabia

E-mail: ahabogal@gmail.com

A. E. Abouelregal

Department of Mathematics, Faculty of Science, Mansoura University, Mansoura 35516, Egypt

E-mail: ahabogal@mans.edu.eg

H. Mohammad-Sedighi- A. H. Shirazi

Mechanical Engineering Department, Faculty of Engineering, Shahid Chamran University of Ahvaz, Ahvaz 61357-43337, Iran

H. Mohammad-Sedighi

Drilling Center of Excellence and Research Center, Shahid Chamran University of Ahvaz, Ahvaz, Iran

E-mail: h.msedighi@scu.ac.ir; hmsedighi@gmail.com

M. Malikan · V. A. Eremeyev ( $\varangle)$

Department of Mechanics of Materials and Structures, Faculty of Civil and Environmental Engineering, Gdansk University of Technology, 80-233 Gdańsk, Poland

E-mail: mohammad.malikan@pg.edu.pl

E-mail: victor.eremeev@pg.edu.pl; victor.eremeev@unica.it

V. A. Eremeyev

DICAAR, Università degli Studi di Cagliari, Via Marengo, 2, 09123 Cagliari, Italy 
Keywords Moore-Gibson-Thompson thermoelasticity · Zakian's computational algorithm $\cdot$ Non-local theory $\cdot$ Half-space $\cdot$ State space approach $\cdot$ Heat source

\section{List of symbols}

\begin{tabular}{ll}
$\lambda, \mu$ & Lame's constants \\
$K$ & Thermal conductivity \\
$\alpha_{t}$ & Thermal expansion coefficient \\
$\boldsymbol{I}$ & Identity tensor \\
$\gamma=(3 \lambda+2 \mu) \alpha_{t}$ & Coupling parameter \\
$\boldsymbol{H}$ & Initial magnetic field \\
$T_{0}$ & Environmental temperature \\
$\boldsymbol{J}$ & Current density \\
$\theta=T-T_{0}$ & Temperature increment \\
$\boldsymbol{u}$ & Displacement vector \\
$T$ & Absolute temperature \\
$\boldsymbol{F}$ & Body force vector \\
$C_{E}$ & Specific heat \\
$Q$ & Heat source \\
$\tau$ & Non-local stress tensor \\
$\tau_{0}$ & Relaxation time \\
$\sigma$ & Local stress tensor \\
$\boldsymbol{h}$ & Induced magnetic field \\
$\epsilon$ & Strain tensor \\
$\rho$ & Material density \\
$\left|x^{\prime}-x\right|$ & Euclidean distance \\
$\boldsymbol{E}$ & Induced electric field \\
$\alpha\left(\left|x^{\prime}-x\right|\right)$ & Non-local Kernel \\
$o_{x y z}$ & Cartesian coordinate \\
$\tau_{i j}$ & Local stress tensor \\
$\nabla^{2}$ & Laplacian operator \\
$\xi=e_{0} a / l$ & Non-local parameter \\
$E$ & Young's modulus \\
$a$ & Internal characteristic length \\
$t$ & Time \\
$l$ & External characteristic length \\
$\mu_{0}$ & Magnetic permeability \\
$e_{0}$ & Adjusting constant \\
$\sigma_{0}$ & Electric conductivity \\
& \\
\hline
\end{tabular}

\section{Introduction}

In-depth investigation on the mechanical and thermal interactions within a solid medium is of great interest in various scientific fields. Modern aeronautical and astronautical engineering, high-energy particle accelerating devices and different systems exploited in nuclear and industrial applications are only a few examples. Consideration of second sound effect in general thermoelasticity models plays a significant role in analyzing elastic body within a verity of scientific and technological fields. In contradiction with physical observation, the infinite thermal propagation speed is predicted through conventional uncoupled theories. On the other hand, general thermoelasticity theories overcome this flaw by supporting second sound phenomenon of heat wave velocity. Biot [1] has made improvements in irreversible thermodynamic processes by implementing improved thermoelastic methods.

By proposing the flux rate concept, Lord and Shulman [2] presented the general model of thermoelasticity. By considering the hyperbolic form of the heat transfer equation, a final rate of thermal signaling is allowed in the model. Another major generalization theory was proposed by Green and Lindsay in the field of coupled 
thermoelasticity [3]. They established temperature-dependent thermoelasticity based on two relaxation times. Except for the heat conduction equation, all the coupled theories changed after the introduction of the mentioned model. Without utilizing Fourier's law, some researchers developed a verity of extensive theories. A significant number of studies have been thoroughly investigated with regard to the non-classical thermoelasticity [4-9].

Green and Naghdi presented a new generalized thermoelastic theory on the basis of Fourier's law [10-12], their theory was proposed in three separate models, the first and second theories are called GN-I and GN-II, respectively, and the third one is called GN-III. Relying on higher-order time derivatives, Abouelregal [13-18] did some investigation in order to alter the conventional Fourier's law. In recent years, many researchers have been attracted to examine the Moore-Gibson-Thompson equation (MGT) as well. This principle is derived based on a cubic differential equation. The method is especially important in the fluid dynamic problems [19]. Quintanilla [20] established a novel thermoelastic heat conduction model based on the Moore-GibsonThompson theorem. Following the introduction of thermal relaxation time in GN-III, Quintanilla [20] proposed the modification of heat equation. Since the beginning of Moore-Gibson-Thompson theory, the number of research works aimed at investigating this theory has considerably increased [21-27]. By implementing the mentioned theories, the mechanical behavior of several structures has been theoretically studied as well [2834].

The size-dependent mechanical behavior of micro/nanostructures is somewhat controversial for the applicability of traditional continuum theory. The classical continuum theory cannot capture the impact of small scale, and therefore, the mechanical behavior of micro/nanostructures cannot be appropriately predicted. Therefore, non-classical continuum theories have been generalized to include the features of micro- and nanomaterials, for instance, the strain gradient theory [35,36], classical couple stress model, non-local elasticity and the modified couple stress theories [37,38].

For miniaturized structures, the size-dependent non-local theory of elasticity should be considered to study the nanomechanical behaviors of such structures like elastic wave dispersion, composite wave propagation, dislocation dynamics, mechanical fractures, surface voltage fluids, etc. In order to deal with the structural problems in small-scale dimensions, Eringen introduced the theory of non-local continuum mechanics in 1972 [39-43]. In recent years, the non-local models were developed to receive increased coverage for the nanoscale systems.

The theories of a non-local elasticity state that the stress at any arbitrary point depends on the strain at other points, whereas the classical continuum mechanics suggests that the stress at a certain location is only related to the strain at that specific local point. In this model, the equilibrium law includes the non-local field residues, and such residues are associated with the constitutive equations in which the stabilization and thermodynamic restriction requirements are balanced. Constituent equations and non-local residues work with gradients of deformation and movements of all body points. Centered on the non-local thermoelasticity theory, Inan and Eringen [44] studied the thermoelastic wave propagation in plates. Wang and Dhaliwal [45] implemented the non-local generalized thermoelasticity energy and showed the presence of a unique solution for the initial and boundary value problems.

Abouelregal and Mohammed [46] introduced a non-local Bernoulli-Euler model and analyzed the thermoelastic interactions in nanoscale beams, using the couple stress and the generalized thermoelasticity models. The theories of Euler-Bernoulli and the modified couple stress have led to discussion by Abouelregal and Marin [47] on non-local nanobeams. The thermal conductivity of the nanoscale beams is also supposed to depend on the temperature changes. Abouelregal [16] introduced a new differential equation system which describes the theory of non-local thermoelasticity with higher derivatives and dual-phase delays. To achieve the proposed theory, he used the Eringen's non-local elasticity and the Taylor expansion methodology of higher-order time derivatives.

The non-local continuum theory expressed by Koutsoumaris et al. [48], either in integral or differential forms, is commonly utilized for describing the size dependency in micro- and nanoscale structures. Liew et al. [49] literary studied the recent works related to the application of non-local elasticity theory for modeling and simulation of graphene sheets. Rajneesh et al. [50] conducted a temporary study on the phase-lagged non-local thermoelastic thick micro-stretches. The non-local theory promotes the solutions for different problems and systems [51-58].

In recent days, more attention has been focused on the analysis of magneto-thermoelasticity problems dealing with the interaction between the magnetic field and the thermomechanical behavior of isotropic and anisotropic materials. Plasma physics is the area in which this theory has excellent examples in nuclear reactors, high power and high-energy gradients, and in also more applications in geophysics. 
With extensive applications such as automotive, nuclear, electronic, military and smart materials and structures, the usage of electromagnetic and magneto-thermoelectric materials has been rapidly enriched [43].

As far as we know, there is hardly an effort to analyze the non-local fractures of the preheated materials, which is extremely important for the handling and/or manufacturing of advanced materials, because the material adjacent to the surface approaches to its melting temperature. In these cases, the theoretical model of non-local effect and fractional order incorporating the amended Fourier law is necessary to establish the thermoelastic responses of micro/nanoscale structures. This paper presents a thermoelastic investigation of a half-space medium based on the Eringen's non-local theory. The studied method demonstrates the temporary non-local thermal stresses in a half-space elastic body. The considered medium is periodically heated and subjected to an external magnetic field. The system of coupled thermoelastic equations is formulated and solved by employing the Laplace transform method. In order to obtain the numerical results for temperature gradients, displacement and non-local stresses in the medium, a numerical Laplace inversion approach using Zakian algorithm [59-61] is then performed. The changes in temperature, displacement and distribution of stresses are explored along the axial direction. Finally, the effects of heat source strength, non-local parameter and the magnet field are considered and discussed.

\section{Non-local MGTE thermoelasticity}

The Fourier's law has been modified by including the relaxation time parameter in the heat flux vector $\vec{q}$ as follows:

$$
\left(1+\tau_{0} \frac{\partial}{\partial t}\right) \vec{q}(\vec{x}, t)=-K \vec{\nabla} \theta(\vec{x}, t)
$$

where $K$ refers to the thermal conductivity, $\theta$ corresponds to the temperature variation, $\tau_{0}$ indicates the positive time lag (relaxation time), and $\vec{x}$ is position vector. Based on GN-III model, the enhanced Fourier's law is expressed as [11]:

$$
\vec{q}(\vec{x}, t)=-\left[K \vec{\nabla} \theta(\vec{x}, t)+K^{*} \vec{\nabla} \vartheta(\vec{x}, t)\right]
$$

where $K^{*}$ is related to the material properties corresponding to the conductivity rate. $\vartheta$ stands for the thermal displacement, and $\dot{\vartheta}=\theta$. Furthermore, the GN-I model is accessible when $K^{*}=0$; similarly, the GN-II model can be obtained by considering $K=0$.

The balancing equation of energy is written by:

$$
\rho C_{E} \frac{\partial \theta}{\partial t}+T_{0} \frac{\partial}{\partial t}\left(\beta_{i j} e_{i j}\right)=-q_{i, i}+Q
$$

where

$$
2 e_{i j}=u_{j, i}+u_{i, j}
$$

in which $C_{E}$ shows the specific heat under the constant strain, $\alpha_{k l}$ symbolizes the components of the tensor of linear thermal expansion, $\beta_{i j}=c_{i j k l} \alpha_{k l}$ are coefficients related to the thermal coupling, $Q$ corresponds to the heat source, and $\rho$ indicates the material's density.

The model described in Eq. (2) has similar issue as for conventional Fourier's theorem, forecasting the infinite spread of thermal wave propagation. Introducing the relaxation time $\tau_{0}$ in the GN-III model, Quintanilla [26] proposed the modified equation of heat conduction. The mentioned equation can be expressed as [20]

$$
\left(1+\tau_{0} \frac{\partial}{\partial t}\right) \vec{q}(\vec{x}, t)=-\left[K \vec{\nabla} \theta(\vec{x}, t)+K^{*} \vec{\nabla} \vartheta(\vec{x}, t)\right]
$$

Conduction equation in linear formation can be achieved by combining Eqs. (3) and (5). The MGTE model is a combination of Lord-Shulman theory [10] and the third version of Green-Naghdi model $[20,30]$

$$
\left(1+\tau_{0} \frac{\partial}{\partial t}\right)\left[\frac{\partial}{\partial t}\left(\rho C_{E} \frac{\partial \theta}{\partial t}\right)+T_{0} \frac{\partial^{2}}{\partial t^{2}}\left(\beta_{i j} e_{i j}\right)-\frac{\partial Q}{\partial t}\right]=\frac{\partial}{\partial t}[\nabla \cdot(K \nabla \theta)]+\frac{\partial}{\partial t}\left[\nabla \cdot\left(K^{*} \nabla \vartheta\right)\right]
$$


Stress tensor is based on the stress at all points of the body according to the Eringen's non-local theory of elasticity [39-41]. The basic equation for elastic and homogeneous materials on the basis of non-local theory in the absence of body forces can be defined as:

$$
\begin{aligned}
\boldsymbol{\tau}(x) & =\int_{V} K\left(\left|\boldsymbol{x}^{\prime}-\boldsymbol{x}\right|, \xi\right) \boldsymbol{\sigma}(x) \mathrm{d} V\left(x^{\prime}\right) \\
\boldsymbol{\varepsilon}\left(x^{\prime}\right) & =\frac{1}{2}\left(\nabla \boldsymbol{u}\left(x^{\prime}\right)+\nabla\left(\boldsymbol{u}^{T}\left(x^{\prime}\right)\right)\right) \\
\boldsymbol{\sigma}\left(x^{\prime}\right) & =\lambda\left(\operatorname{div} \boldsymbol{u}\left(x^{\prime}\right)\right) I+2 \mu \boldsymbol{\varepsilon}\left(x^{\prime}\right)-\gamma \theta\left(x^{\prime}\right) \boldsymbol{I}
\end{aligned}
$$

The non-local kernel $K\left(\left|\boldsymbol{x}^{\prime}-\boldsymbol{x}\right|\right)$ describes the correlation of stress at point $\boldsymbol{x}$ on the stress in $\boldsymbol{x}^{\prime}$-point of the elastic body. Also, $\xi$ relies on the material constant $\left(e_{0} a / l\right)$. The parameter $a$ denotes the lattice parameter (granular distance, internal characteristic length and length of $\mathrm{C}-\mathrm{C}$ bonds), and $l$ indicates the crack length (external characteristic length), while $e_{0}$ stands for the constant length for each specific material.

By employing the Eringen's assumptions, the differential form of the non-local stress tensor $\tau$ can be expressed as

$$
\left(1-\xi^{2} \nabla^{2}\right) \tau=\sigma
$$

Equation (10) considers the small-size effects for the physiomechanical behavior of nanostructures. It should be noticed that if the internal length-scale parameter $a$ is ignored, $\xi=e_{0} a / l$ is equal to zero, and then, the constitutive relation of classical elasticity theory can be obtained from Eq. (10).

The linear momentum equilibrium leads to the following equation of motion:

$$
\nabla \cdot \tau+\boldsymbol{F}=\rho \ddot{u},
$$

By adopting Eqs. (10) and (11), the invariant form of the non-local equation of motion can be derived as follows:

$$
\nabla \cdot \boldsymbol{\sigma}+\left(1-\xi^{2} \nabla^{2}\right) \boldsymbol{F}=\rho\left(1-\xi^{2} \nabla^{2}\right) \ddot{u} .
$$

In terms of temperature and displacements, the governing equations are achieved by:

$$
(\lambda+\mu) \nabla(\nabla \boldsymbol{u})+\mu \nabla^{2} \boldsymbol{u}-\gamma \nabla \theta+\left(1-\xi^{2} \nabla^{2}\right) \boldsymbol{F}=\rho\left(1-\xi^{2} \nabla^{2}\right) \ddot{u} .
$$

The Maxwell's electromagnetic field equations for a homogenous thermoelastic conducting body are defined as [62]

$$
\begin{gathered}
\boldsymbol{J}=\nabla \times \boldsymbol{h}, \nabla \times \boldsymbol{E}=-\mu_{0} \frac{\partial \boldsymbol{h}}{\partial t}, \boldsymbol{J}=\sigma_{0}\left(\boldsymbol{E}+\mu_{0}\left(\frac{\partial \boldsymbol{u}}{\partial t} \rtimes \boldsymbol{H}\right)\right), \\
\boldsymbol{h}=\nabla \times(\boldsymbol{u} \rtimes \boldsymbol{H}), \nabla \cdot \boldsymbol{h}=0,
\end{gathered}
$$

\section{Formulation of the problem}

Under the influence of a periodically varying heat source spread out over the plane surface along the $x$-direction, an infinitely isotropic solid initially at the temperature $T_{0}$ is considered here (see Fig. 1). The one-dimensional disturbance of the medium is also taken into consideration. Hence, the displacement vector $\boldsymbol{u}$ can be defined in the following form:

$$
u_{x}=u(x, t), u_{y}=u_{z}=0 .
$$

The impact of the longitudinal magnetic field with a constant strength is presumed to act perpendicular to the $\mathbf{z}$-axis, i.e., $\boldsymbol{H}=\left(0, H_{x}, 0\right)$. The Lorentz force can be derived by using the Maxwell's equations as $\boldsymbol{F}=\boldsymbol{J} \rtimes \boldsymbol{H}$; therefore, the equation of motion leads to:

$$
\boldsymbol{F}=\left(f_{x}, f_{y}, f_{z}\right)=-\sigma_{0} \mu_{0} H_{x}^{2}\left(\frac{\partial \boldsymbol{u}}{\partial t}, 0,0\right) .
$$

For the one-dimensional case, the constitutive relation (10) becomes

$$
\left(1-\xi^{2} \frac{\partial^{2}}{\partial x^{2}}\right) \tau_{x x}=(\lambda+2 \mu) \frac{\partial \boldsymbol{u}}{\partial x}-\gamma \theta
$$


Using Eqs. (16) and (17), the equation of motion (13) can be reformed as follows:

$$
(\lambda+2 \mu) \frac{\partial^{2} u}{\partial x^{2}}-\gamma \frac{\partial \theta}{\partial x}-\sigma_{0} \mu_{0} H_{x}^{2}\left(1-\xi^{2} \frac{\partial^{2}}{\partial x^{2}}\right) \frac{\partial u}{\partial t}=\rho\left(1-\xi^{2} \frac{\partial^{2}}{\partial x^{2}}\right) \frac{\partial^{2} u}{\partial t^{2}} .
$$

The generalized MGTE heat equation will be stated in the following form [20,30]

$$
\left(1+\tau_{0} \frac{\partial}{\partial t}\right)\left[\rho C_{E} \frac{\partial^{2} \theta}{\partial t^{2}}+\gamma T_{0} \frac{\partial^{2}}{\partial t^{2}}\left(\frac{\partial u}{\partial x}\right)-\frac{\partial Q}{\partial t}\right]=\left(K \frac{\partial}{\partial t}+K^{*}\right) \frac{\partial^{2} \theta}{\partial x^{2}} .
$$

The following non-dimensional variables are introduced

$$
\begin{gathered}
\left\{x^{\prime}, u^{\prime}\right\}=c_{0} \omega_{0}\{x, u\},\left\{t^{\prime}, \tau_{0}^{\prime}\right\}=c_{0}^{2} \omega_{0}\left\{t, \tau_{0}\right\}, \xi^{\prime}=c_{0}^{2} \omega_{0}^{2} \xi, \\
\theta^{\prime}=\frac{\theta}{T_{0}}, \sigma_{x}^{\prime}=\frac{\sigma_{x}}{\mu}, Q^{\prime}=\frac{Q}{K T_{0} c_{0}^{2} \omega_{0}^{2}}, c_{0}^{2}=\frac{(\lambda+2 \mu)}{\rho}, \omega_{0}=\frac{\rho C_{E}}{K},
\end{gathered}
$$

The parameter $c_{0}$ indicates the velocity propagation of the isothermal elastic wave. The governing equations (17)-(19) take the following form after using the above-mentioned non-dimensional variables (removing the primes)

$$
\begin{aligned}
& \left(1-\xi^{2} \frac{\partial^{2}}{\partial x^{2}}\right) \tau_{x x}=\beta^{2} \frac{\partial u}{\partial x}-b \theta \\
& \left(1-\xi^{2} \frac{\partial^{2}}{\partial x^{2}}\right) \frac{\partial^{2} u}{\partial t^{2}}=\frac{\partial^{2} u}{\partial x^{2}}-\frac{b}{\beta^{2}} \frac{\partial \theta}{\partial x}-\varepsilon\left(1-\xi^{2} \frac{\partial^{2}}{\partial x^{2}}\right) \frac{\partial u}{\partial t}, \\
& \left(1+\tau_{0} \frac{\partial}{\partial \mathrm{t}}\right)\left[\frac{\partial \theta}{\partial t}+g \frac{\partial}{\partial t}\left(\frac{\partial u}{\partial x}\right)-Q\right]=\left(\frac{\partial}{\partial t}+K_{0}^{*}\right) \frac{\partial^{4} \theta}{\partial x^{4}},
\end{aligned}
$$

where

$$
\beta^{2}=\frac{(\lambda+2 \mu)}{\mu}, b=\frac{\gamma T_{0}}{\mu}, \varepsilon=\frac{\sigma_{0} \mu_{0} H_{x}^{2}}{\rho \mathrm{c}_{0}^{2} \omega_{0}}, g=\frac{\gamma}{\rho C_{E}}, K_{0}^{*}=\frac{K^{*}}{K c_{0}^{2} \omega_{0}} .
$$

To further study the problem, governing Eqs. (21)-(23) should be solved and the distributions of displacement $(u)$, temperature $(\theta)$ and non-local thermal stress $\left(\tau_{x x}\right)$ must be determined within the medium. The following initial conditions are assumed to deal with the current problem:

$$
\begin{aligned}
& \theta(x, 0)=\frac{\partial \theta(x, 0)}{\partial t}=0=u(x, 0)=\frac{\partial u(x, 0)}{\partial t} \\
& u(\mathrm{x}, t) \rightarrow 0, \theta(\mathrm{x}, t) \rightarrow 0 \text { as } x \rightarrow \infty
\end{aligned}
$$

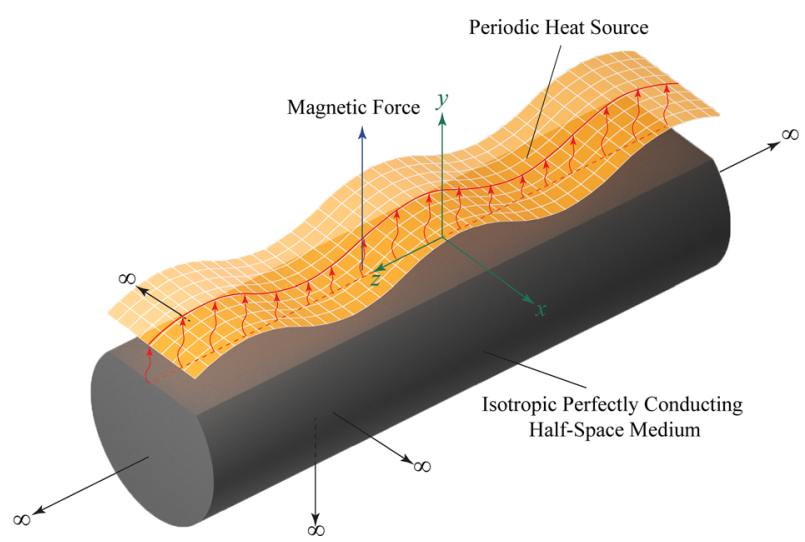

Fig. 1 Schematic of the half-space under the influence of external magnetic field and periodical heat source 
Due to the periodic heat source $Q(x, t)$ of constant intensity $Q_{0}$, the surface $x=0$ of the half-space is heated. The following non-dimensional form is assumed for the heat source [63]

$$
Q(x, t)=\left\{\begin{array}{l}
Q_{0} \delta(x) \sin \left(\pi t / t_{0}\right), \begin{array}{c}
0 \leq t \leq t_{0} \\
0,
\end{array} \\
t>t_{0}
\end{array}\right.
$$

in which the parameter $t_{0}$ is constant and the function $\delta(x)$ denotes the Dirac's delta function.

\section{Solution in the Laplace transform domain}

By utilizing the Laplace transform approach, the transforming Eqs. (24)-(26) are described by the relationship:

$$
\bar{f}(x, t)=\int_{0}^{\infty} f(x, t) e^{-s t} \mathrm{~d} t,
$$

and then, using the initial conditions (25) yields:

$$
\begin{array}{r}
\left(1-\xi^{2} \frac{\mathrm{d}^{2}}{\mathrm{~d} x^{2}}\right) \bar{\tau}_{x x}=\frac{\mathrm{d} \bar{u}}{\mathrm{~d} x}-\bar{\theta} \\
\left(\mathrm{s}+K_{0}^{*}\right) \frac{\mathrm{d}^{2} \bar{\theta}}{\mathrm{d} x^{2}}=s\left(1+\mathrm{s} \tau_{0}\right)\left[s \bar{\theta}+\mathrm{s} \varepsilon \frac{\mathrm{d} \bar{u}}{\mathrm{~d} x}-\frac{\pi t_{0} \mathrm{Q}_{0}}{\pi^{2}+s^{2} t_{0}^{2}} \delta(x)\right] \\
s^{2}\left(1-\xi^{2} \frac{\mathrm{d}^{2}}{\mathrm{~d} x^{2}}\right) \bar{u}=\frac{\mathrm{d}^{2} \bar{u}}{\mathrm{~d} x^{2}}-\frac{\mathrm{d} \bar{\theta}}{\mathrm{d} x}-s g\left(1-\xi^{2} \frac{\mathrm{d}^{2}}{\mathrm{~d} x^{2}}\right) \bar{u}
\end{array}
$$

The differential equations presented in Eq. (30) can be rewritten in the matrix-vector form by $[64,65]$ :

$$
\frac{\mathrm{d} \boldsymbol{V}(x, s)}{\mathrm{dx}}=A(s) \boldsymbol{V}(x, s)+f(x, s),
$$

with

$$
\boldsymbol{V}(x, s)=\left(\begin{array}{c}
\bar{\theta} \\
\bar{u} \\
\mathrm{~d} \bar{\theta} \\
\frac{\mathrm{d} x}{\mathrm{~d} \bar{u}} \\
\mathrm{~d} x
\end{array}\right), A(s)=\left(\begin{array}{cccc}
0 & 0 & 1 & 0 \\
0 & 0 & 0 & 1 \\
\alpha_{4} & 0 & 0 & \alpha_{5} \\
0 & \frac{\alpha_{2}}{\alpha_{1}} & \frac{\alpha_{3}}{\alpha_{1}} & 0
\end{array}\right), f(x, s)=\left(\begin{array}{c}
0 \\
0 \\
-\alpha_{6} \delta(x) \\
0
\end{array}\right),
$$

and

$$
\begin{gathered}
\alpha_{1}=1+s \xi^{2}(s+g), \alpha_{2}=s(s+g), \alpha_{4}=\frac{s^{2}\left(1+\mathrm{s} \tau_{0}\right)}{\left(\mathrm{s}+K_{0}^{*}\right)}, \\
\alpha_{5}=\frac{s^{2} \varepsilon\left(1+\mathrm{s} \tau_{0}\right)}{\left(\mathrm{s}+K_{0}^{*}\right)}, \alpha_{6}=\frac{\pi t_{0} \mathrm{Q}_{0} s\left(1+\mathrm{s} \tau_{0}\right)}{\left(\pi^{2}+s^{2} t_{0}^{2}\right)\left(\mathrm{s}+K_{0}^{*}\right)} .
\end{gathered}
$$

The solutions for $\bar{u}(x)$ and $\bar{\theta}(x)$ can be interpreted by using the eigenvalue approach discussed in [64,65]. Applying the eigenvalue approach to the system (31) under the regular condition (29) results in:

$$
\begin{aligned}
& \bar{u}(x, s)=\frac{\alpha_{6}\left(\mathrm{e}^{-k_{2} x}-\mathrm{e}^{-k_{1} x}\right)}{2\left(k_{1}^{2}-k_{2}^{2}\right)} \\
& \bar{\theta}(x, s)=\frac{\alpha_{6}\left(k_{1}\left(\alpha_{2}-\alpha_{1} k_{2}^{2}\right) \mathrm{e}^{-k_{2} x}-k_{2}\left(\alpha_{2}-\alpha_{1} k_{1}^{2}\right) \mathrm{e}^{-k_{1} x}\right)}{2 k_{1} k_{2}\left(k_{1}^{2}-k_{2}^{2}\right)}
\end{aligned}
$$

The parameters $k_{1}^{2}$ and $k_{1}^{2}$ indicate the roots of the following equation with positive real parts:

$$
k^{4}-m_{1} k^{2}+m_{2}=0,
$$

where the coefficients $m_{1}$ and $m_{2}$ satisfy the equations

$$
m_{1}=\alpha_{4}+\frac{\alpha_{2}}{\alpha_{1}}+\frac{\alpha_{5}}{\alpha_{1}}, m_{2}=\frac{\alpha_{2} \alpha_{4}}{\alpha_{1}}
$$


Table 1 Parameters $\alpha_{i}$ and $K_{i}$ for $N=5$ [66]:

\begin{tabular}{lll}
\hline$j$ & Material properties & Value \\
\hline 1 & $12.83767675+666063445 i$ & $-36902.08210+196990.4257 i$ \\
2 & $212.22613209+5.012718792 i$ & $61277.02524-95408.62551 i$ \\
3 & $310.93430308+8.409673116 i$ & $-28916.56288+18169.18531 i$ \\
4 & $48.776434715+11.92185389 i$ & $4655.361138-1.901528642 i$ \\
5 & $55.225453361+15.72952905 i$ & $-118.7414011-141.3036911 i$ \\
\hline
\end{tabular}

Substituting from Eqs. (37) and (38) into (32), the non-local stress $\bar{\tau}_{x x}$ is described by:

$$
\begin{gathered}
\bar{\tau}_{x x}(x, s)=\frac{\alpha_{6} k_{1} \mathrm{e}^{-k_{1} x}}{2\left(k_{1}^{2}-k_{2}^{2}\right)\left(1-\xi^{2} k_{1}^{2}\right)}-\frac{\alpha_{6} k_{2} \mathrm{e}^{-k_{2} x}}{2\left(k_{1}^{2}-k_{2}^{2}\right)\left(1-\xi^{2} k_{2}^{2}\right)}+ \\
\frac{\alpha_{6} k_{2}\left(\alpha_{2}-\alpha_{1} k_{1}^{2}\right) \mathrm{e}^{-k_{1} x}}{2 k_{1} k_{2}\left(k_{1}^{2}-k_{2}^{2}\right)\left(1-\xi^{2} k_{1}^{2}\right)}-\frac{\alpha_{6} k_{1}\left(\alpha_{2}-\alpha_{1} k_{2}^{2}\right) \mathrm{e}^{-k_{2} x}}{2 k_{1} k_{2}\left(k_{1}^{2}-k_{2}^{2}\right)\left(1-\xi^{2} k_{2}^{2}\right)}
\end{gathered}
$$

The solution of the strain $\bar{e}(x, s)$ can be expressed as:

$$
\bar{e}(x, s)=\frac{\mathrm{d} \bar{u}(x, s)}{\mathrm{d} x}=\frac{\alpha_{6}\left(k_{1} \mathrm{e}^{-k_{1} x}-k_{2} \mathrm{e}^{-k_{2} x}\right)}{2\left(k_{1}^{2}-k_{2}^{2}\right)}
$$

An empirical reversal of a Laplace domain solution is almost difficult to achieve, and therefore, a numerical reversal approach is required. Several computational algorithms in the literature can be exploited to carry out the inversion of Laplace transform. Each approach has its own application and can be utilized for a specific purpose. The Zakian approximation technique [59-61] is employed here to obtain the numerical results and evaluate the variables in time domain.

\section{Zakian's method}

The obtained solution for the physical fields described in (37), (38), (41) and (42) is transformed into the Laplace domain using the techniques of numerical inversion in the physical domain. Zakian's technique considers the time domain with the following infinite number of evaluations using the weighted domain function [59-61]:

$$
f(x, t)=\frac{2}{t} \sum_{i=1}^{N} \operatorname{Re}\left\{K_{i} \bar{F}\left(\frac{\alpha_{i}}{t}\right)\right\}
$$

One of the most important features of the introduced method is that it can be easily used and applied in different problems. The conjugate pairs may be real or complex in the formula $K_{i}$ and $\alpha_{i}$. The parameter $N$ indicates the number of assumed terms which means the truncation from the endless sequence and can be optimized or selected in accordance with the considered area. Table 1 includes the constants $K_{i}$ and $\alpha_{i}$ for $N=5$. Only one free parameter $N$ can be specified.

\section{Special cases}

We can study various theories on the introduced system of equations and the considered model.

\subsection{Generalized theories of thermoelasticity}

Calculations for the generalized models of thermoelasticity are performed by assuming $\xi=0$.

- The coupled thermoelasticity theory (CTE) can be obtained by setting $\tau_{0}=K=K^{*}=0$.

- The generalized theory of thermoelasticity with relaxation time $(\mathbf{L S})$ can be achieved when $\tau_{0}>0$ and taking $K^{*}=0$. 
- The generalized Green-Naghdi theory of type II (GN-II) can obtained by assuming $\tau_{0}=0$ and putting $K=0$.

- The generalized Green-Naghdi theory of type III (GN-III) is accessible by adopting $\tau_{0}=0$ and $K, K^{*}>0$.

- The generalized Moore-Gibson-Thompson thermoelasticity (MGTE) is available when $\tau_{0}, K, K^{*}>0$.

\subsection{Non-local theories of thermoelasticity}

The computations for the non-local models of thermoelasticity are performed when $\xi>0$.

- The non-local classical theory of thermoelasticity (NCTE) can be obtained by setting $\tau_{0}=K=K^{*}=0$.

- The non-local generalized thermoelasticity theory with relaxation time (NLS) can be obtained when $\tau_{0}>0$ and taking into account $K^{*}=0$.

- The non-local generalized Green-Naghdi theory of type II (NGN-II) is available by assuming $\tau_{0}=0$ and putting $K=0$.

- The non-local generalized Green-Naghdi theory of type III (NGN-III) is valid by assuming $\tau_{0}=0$ and putting $K, K^{*}>0$.

- The non-local Moore-Gibson-Thompson thermoelasticity (NMGTE) is accessible when $\tau_{0}, K, K^{*}>0$.

\section{Numerical results}

Numerical results are now presented to explain the variation of displacement $u$, temperature $\theta$, the strain $e$ and the non-local thermal stress $\tau_{x x}$ as well as discussed on the obtained results. In order to find the solutions for physical field in space-time areas, we must apply a technique of inversion approximation (40) proposed by Zakian [16,48] to equations (34), (35), (38) and (39), respectively. For all numerical calculations, Mathematica programming Language is employed. The material domain is also defined as copper for numerical calculations. The mechanical and thermoelastic physical parameters corresponding to the mentioned material are given by [67].

$$
\begin{gathered}
K=386 \mathrm{Wm}^{-1} \mathrm{~K}^{-1}, C_{E}=384.56 \mathrm{~J} / \mathrm{kgK}, \alpha_{\mathrm{t}}=1.78 \times 10^{-5} \mathrm{~K}^{-1}, \\
E=128 \mathrm{GPa}, T_{0}=293 \mathrm{~K}, \rho=8954 \mathrm{kgm}^{-3}, \sigma_{0}=10^{-9} / 36 \pi \mathrm{Fm}^{-1}, \\
t=0.2 \mathrm{~s}, \mu_{0}=4 \pi \times 10^{-7} \mathrm{H} \mathrm{m}^{-1}, H_{\mathrm{x}}=10^{-7} / 4 \pi \mathrm{Am}^{-1}, v=0.36 .
\end{gathered}
$$

For different values of the non-local parameter $\xi$, heat source intensity and magnetic field $H_{x}$, the numerical calculations of the field variables have been made. The findings are graphically analyzed at various locations through Figs. 2, 3, 4, 5, 6, 7, 8, 9, 10, 11, 12 and 13, with an average range of 0-10. Results show that the field variables, e.g., $u, \theta, e$, and the non-local distribution of thermal stress $\left(\tau_{x x}\right)$ depend not only on the time $t$ and the space coordinate $x$ but also on the strength of the heat source $Q_{0}$, the relaxation time $\tau_{0}$, the non-local parameter $\xi$, the material parameter $K^{*}$ and of course the magnetic field $H_{x}$. Discussion on the numerical results and the illustrative examples are divided into three sections.

\subsection{The effect of non-local parameter}

In order to accurately design the small-scale devices, it is vital to have the adequate understanding of nonclassical behavior of such systems. In the last few years, great attentions have been focused on the static and dynamic behavior of nanoscale structures. This subsection aims at investigating the bending, vibration and wave propagation of nanostructures along the lines of the theory of non-local elasticity.

Our findings are presented in the form of figures to study the effect of non-local parameter $\xi$ on the temperature, displacement, strain and the non-local thermal stress (Figs. 2, 3, 4, 5). It is noted that by assuming $\xi=0$, the classical models of thermoelasticity are adopted, while the non-local theories of elasticity and thermoelasticity are indicated by some assigned values $(\xi=0.1,0.3)$. In this case, the values $\tau_{0}=0.02$, $Q_{0}=1$ and $\varepsilon=1$ have been taken into account. Figures 2, 3, 4 and 5 indicate the significant impact of the parameter $\xi$ on different fields of the current problem.

Figure 2 exhibits the displacement difference $u$ against position $x$ for different values of $\xi$. From this figure, it is observed that the displacement magnitude first increases with distance very quickly, reaches the 


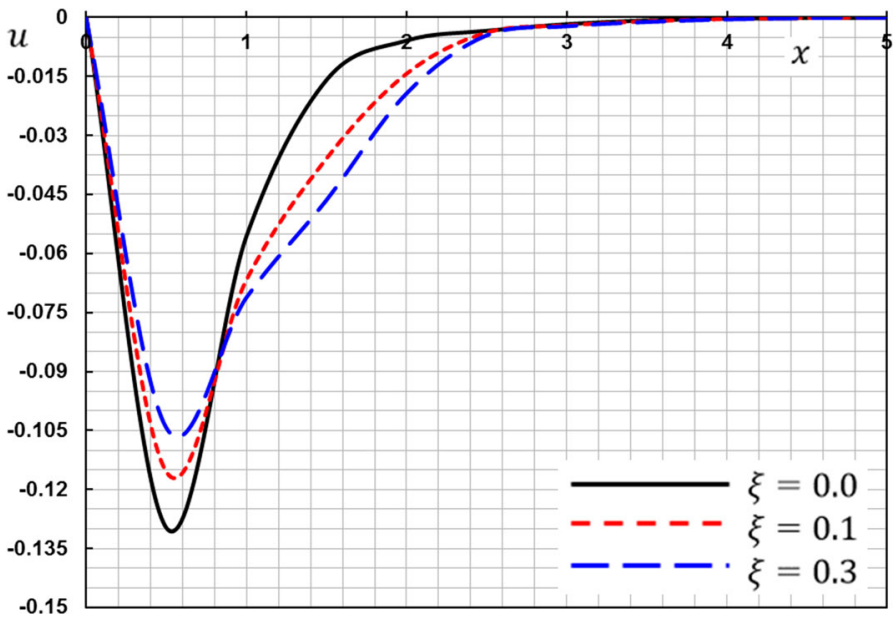

Fig. 2 The variation of the displacement $u$ with $\xi$

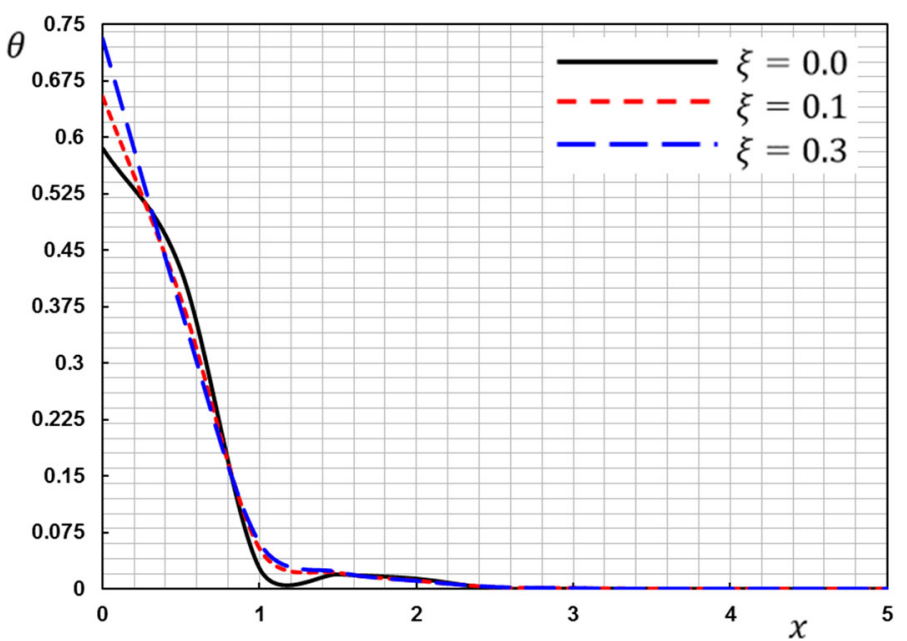

Fig. 3 The variation of the temperature $\theta$ with $\xi$

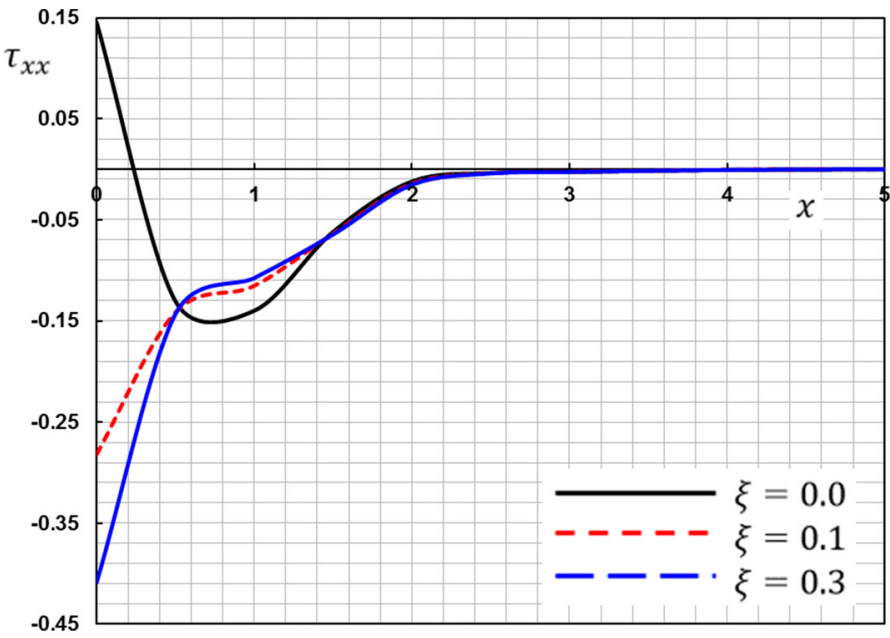

Fig. 4 The variation of the non-local stress $\tau_{x x}$ with non-local parameter $\xi$ 


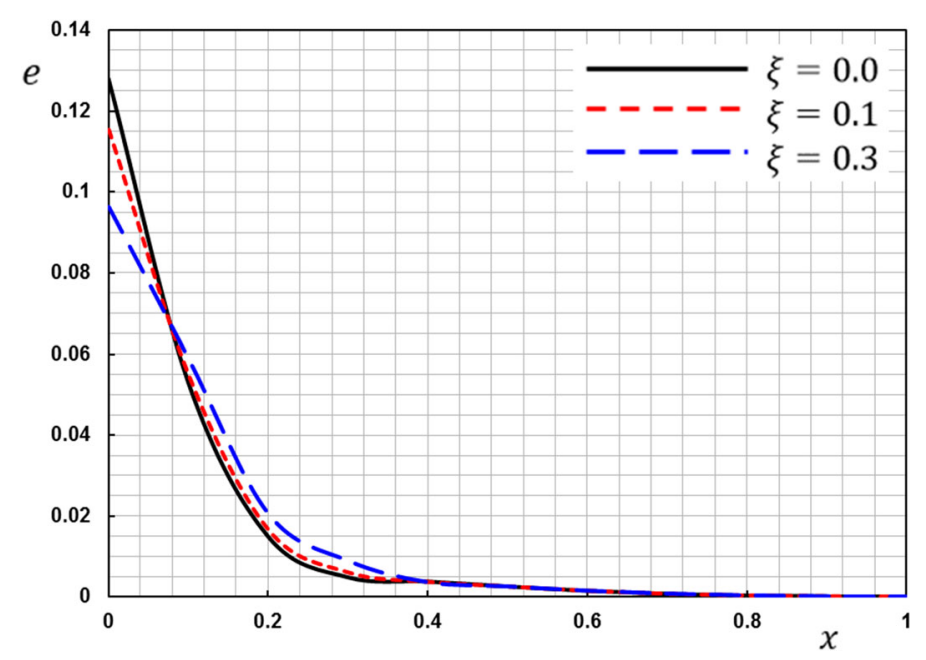

Fig. 5 The variation of the strain $e$ with non-local parameter $\xi$

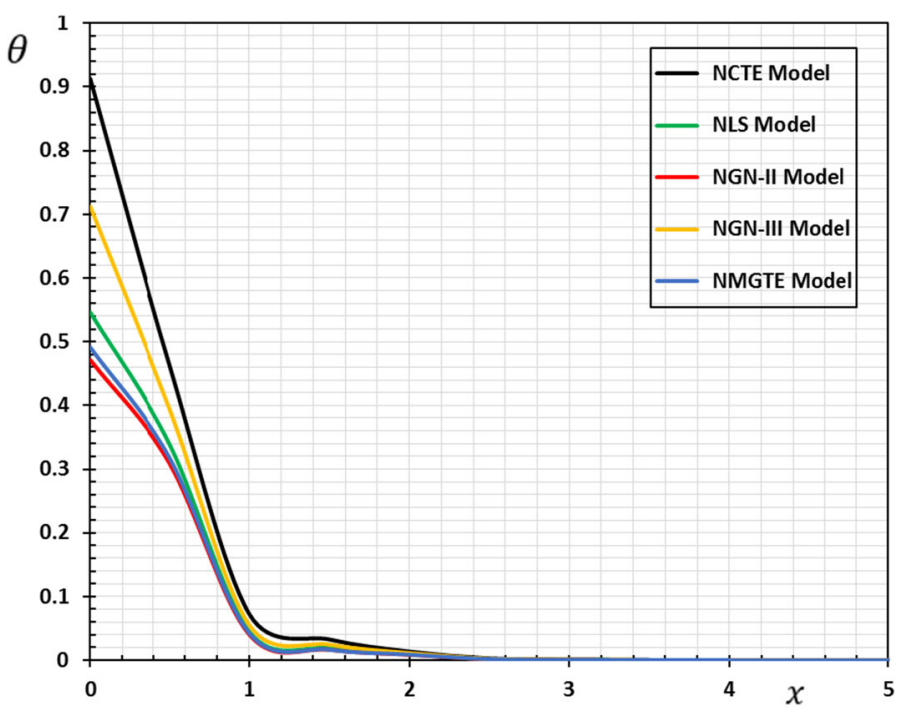

Fig. 6 Field variable $\theta$ for different non-local thermoelasticity theories

maximum value and then decays to zero for $x \geq 3$. The graph 2 also depicts the negative displacement values due to the fact that the heat source changes periodically over the time (a short time active sinus pulse). It is also concluded that the point of displacement decreases with the value of parameter $\xi$ and the effect of parameter $\xi$ is considerable in the interval $0 \leq x \leq 2$.

The variation of the temperature $\theta$ against the distance $x$ is shown in Fig. 3 to examine the impact of $\xi$ on the temperature in the context of MGTE theorem. Figure 3 reveals that the values of temperature distribution $\theta$ increase with an increase in the parameter $\xi$ in the ranges $0 \leq x \leq 0.3$ and $0.8 \leq x \leq 1.2$, reduce in the range of $0.3 \leq x \leq 0.8$ and then finally become nearly stationary as $x>2.4$. This is due to the fact that with the short period of time, the heat source varies periodically as demonstrated in [65-69]. 


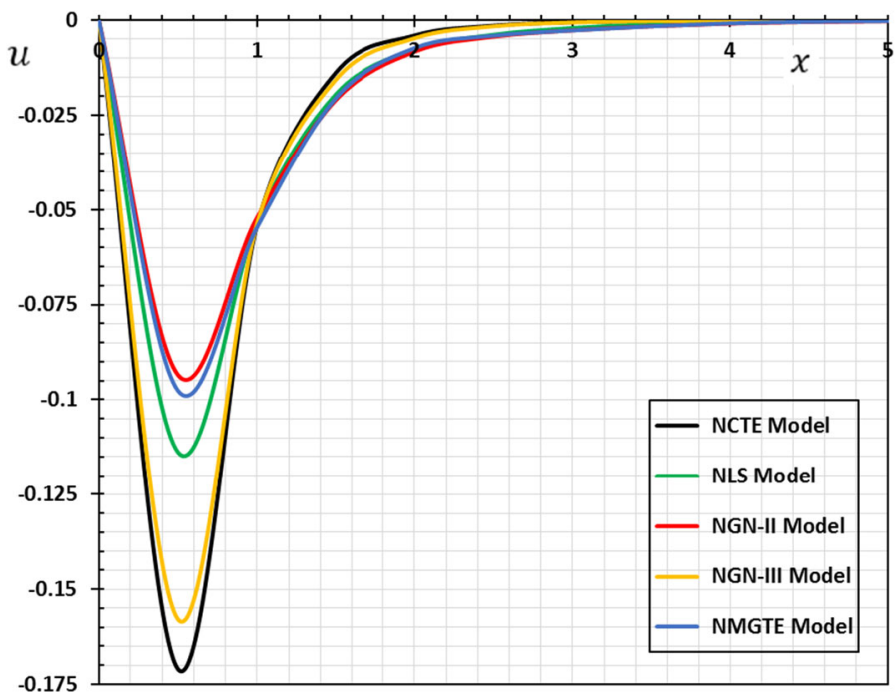

Fig. 7 Displacement $u$ for different non-local thermoelasticity theories

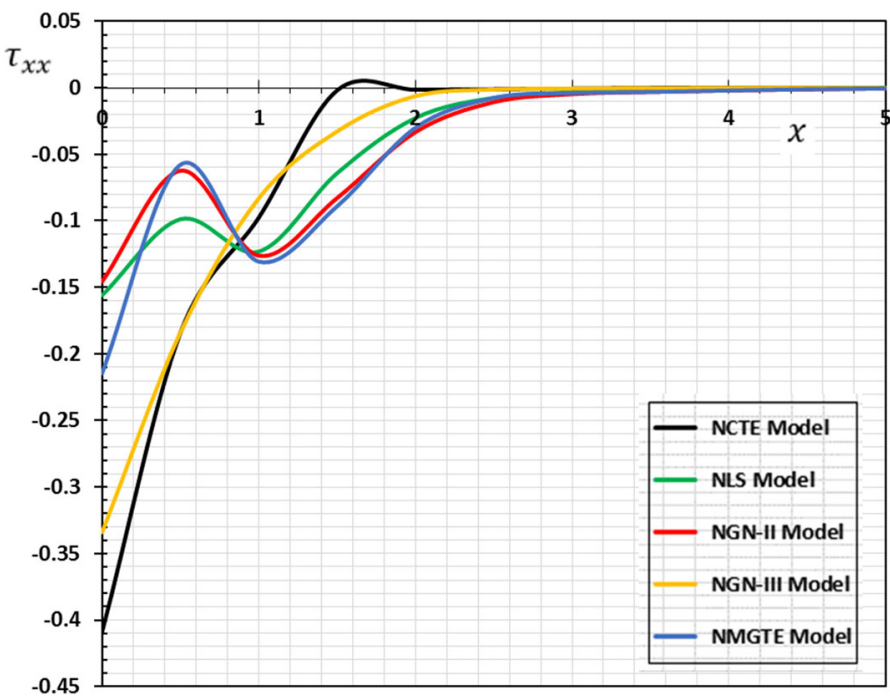

Fig. 8 The non-local stress $\tau_{x x}$ for different non-local thermoelasticity theories

Figure 4 indicates the change of non-local stress increment $\tau_{x x}$ with respect to the distance $x$. The non-local stress value for $\xi=0.0,0.1,0.3$ is adversely affected, and as the parameter $\xi$ decreases, the stress magnitude increases. The influence of the existence of heat sources distributed throughout the plane region $x=0$ will imply these types of activity in all field variables. Stress and displacement fields are observed to be compressive first and then begin to decay in size and finally approach to zero in all cases. The maximum stress $\tau_{x x}$ is close to the boundary of the half-space, and $\tau_{x x}$ is highly affected by the values of parameter $\xi$, as indicated in [69] and shown in Fig. 4.

Figure 5 presents the strain variation $e$ for different values of the parameter $\xi$ versus distance $x$. This figure illustrates that the strain $e$ decreases as the distance is increased and eventually approaches to zero. It is noted from the figure that the strain increases with increasing the parameter $\xi$ in some periods and decreases in the remaining periods. This may be because of the periodically changes of heat source over time.

From the results and previous discussion, it is found that the microscale effect is not apparent for structures with dimensions in the order of microns, while it can be noticeable in nanoscale dimensions which is consistent with the observations of Wang and Liew [70]. Also, when the stress at the source of a nanoscale heating problem is determined, the non-local behavior is a key factor which cannot be ignored [16,48-50]. According to this new non-local theory, we must define a new classification for all materials in accordance with the elastic non- 


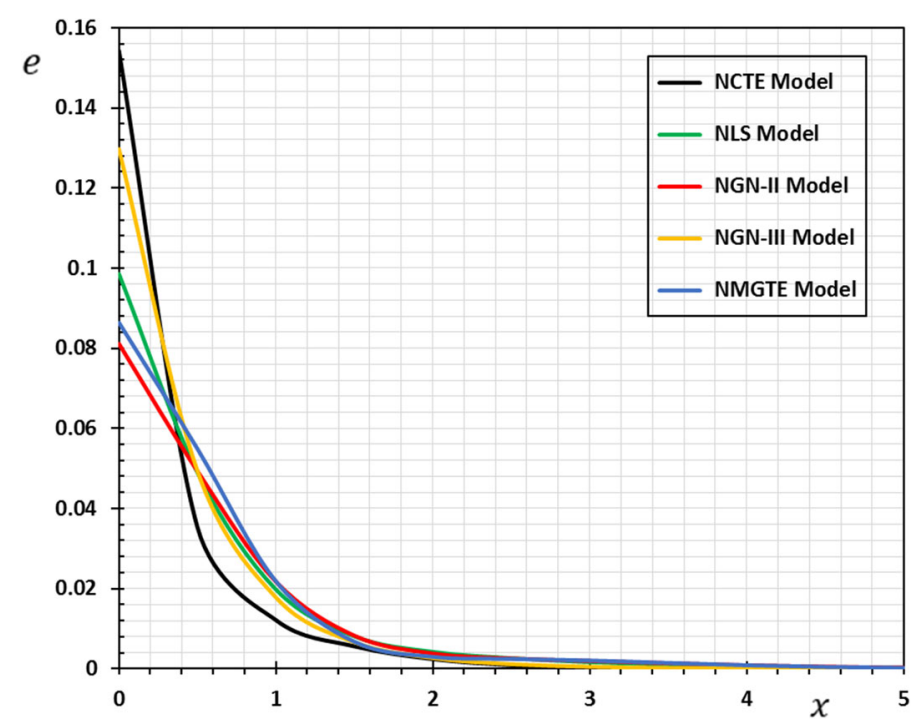

Fig. 9 The strain $e$ for different non-local thermoelasticity theories

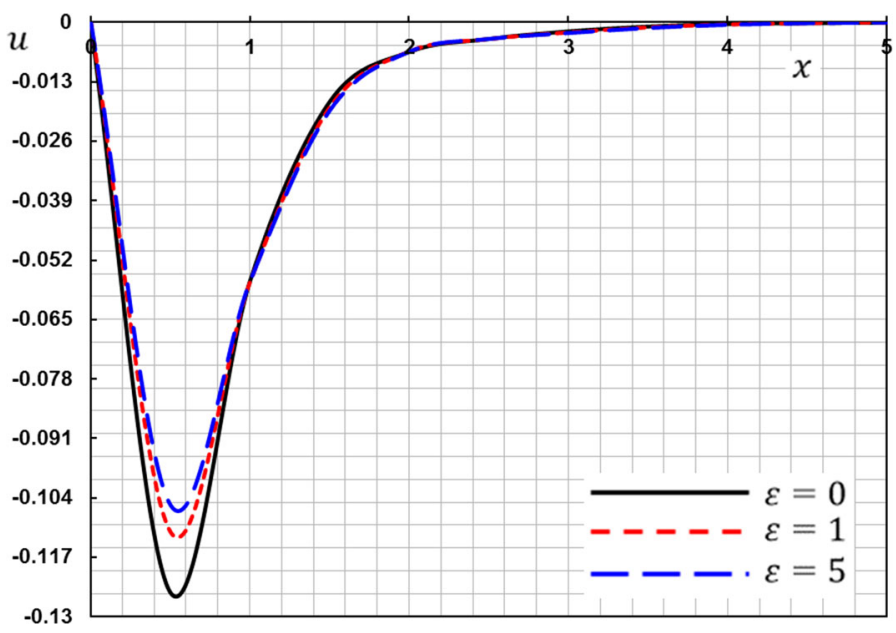

Fig. 10 The displacement $u$ under the effect of applied magnetic field

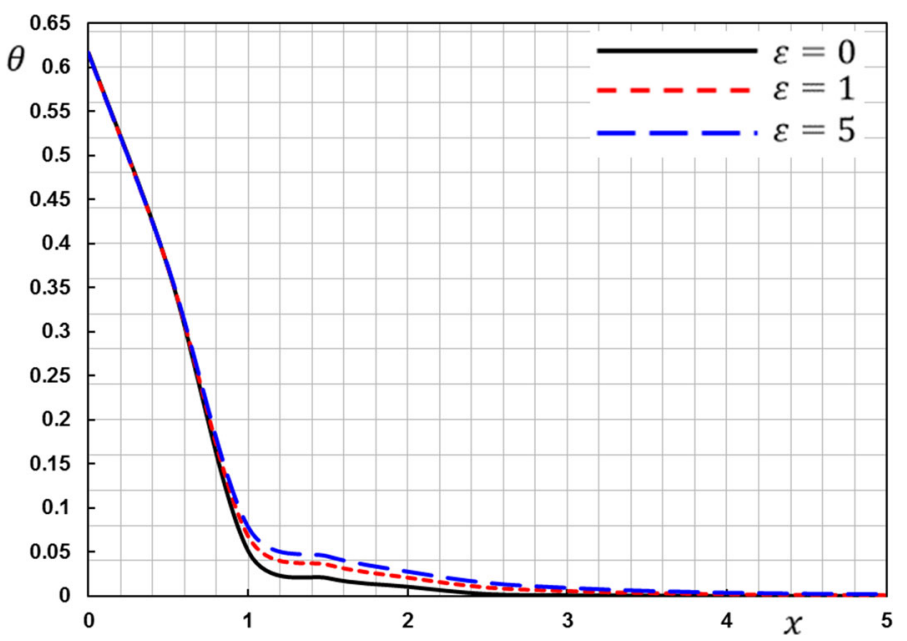

Fig. 11 The temperature $\theta$ under the effect of applied magnetic field 


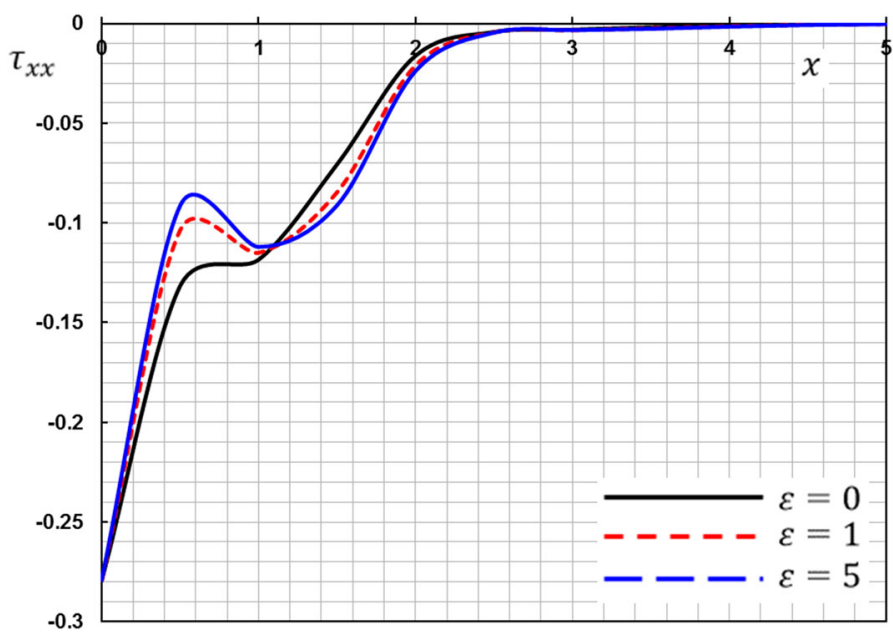

Fig. 12 The non-local stress $\tau_{x x}$ under the effect of applied magnetic field

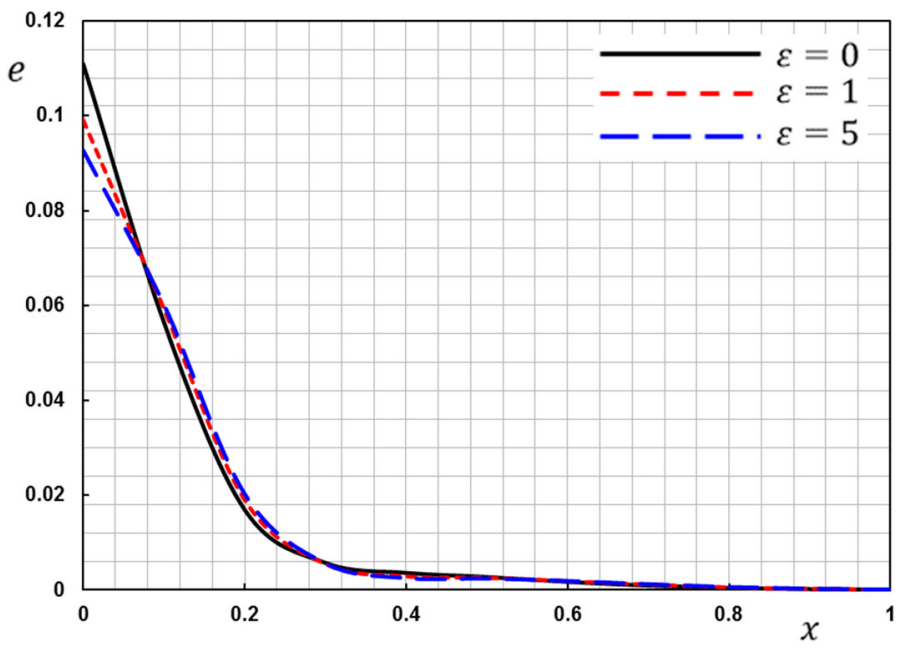

Fig. 13 The strain $e$ under the influence of applied magnetic field

locality parameter $\xi$. The field variables often disappear with large $x$ values which is the basic characteristic of generalized thermoelasticity. This emphasizes that the thermally disrupted area is for a certain moment limited. The proposed theory has now been validated physically. It is worth mentioning that the heat waves propagate at infinite spreading rates in the coupled theory, rather than a finite rate in the generalized context. In the coupled theory, the solution immediately fills the entire medium and therefore is not zero for any small value of time (although it may be very small). However, the solution obtained with the general thermoelasticity equations shows the conduct of wave propagation at limited rates.

\subsection{Comparison of different thermoelasticity models}

In this subsection, it is aimed to clarify the current thermoelastic non-local theory based on the MGTE heat transfer model. The non-dimensional field variables against the space variable $x$ are studied for different thermoelasticity theories, while the non-local parameter $\xi$ is assumed to be fixed $(\xi=0.1)$. The comparisons between the changes in the considered fields under the theories of non-local thermoelasticity (NCTE, NLS, NGN-II, NGN-III models and NMGTE) are depicted in Figs. 6, 7, 8 and 9 and presented in Tables 2, 3, 4 and 5. The tables can be used for comparative studies for future researches and practical studies. 
Table 2 The temperature $\theta$ against distance $x$

\begin{tabular}{llllll}
\hline$x$ & NCTE & NLS & NGN-II & NGN-III & NMGTE \\
\hline 0.0 & 0.913099 & 0.546272 & 0.471496 & 0.712558 & 0.49124 \\
0.5 & 0.464366 & 0.339959 & 0.309865 & 0.395407 & 0.318432 \\
1.0 & 0.0726438 & 0.0449211 & 0.0403095 & 0.0553329 & 0.0423645 \\
1.5 & 0.0326925 & 0.0190259 & 0.0164673 & 0.0246476 & 0.016466 \\
2.0 & 0.0136876 & 0.00956508 & 0.00864791 & 0.011342 & 0.0088852 \\
2.5 & 0.00298238 & 0.00189095 & 0.00169282 & 0.00232006 & 0.0017586 \\
3.0 & 0.00115897 & 0.000708719 & 0.000622444 & 0.000894157 & 0.000629663 \\
3.5 & 0.000436966 & 0.000296909 & 0.000267179 & 0.000356188 & 0.000274229 \\
4.0 & 0.000112863 & $7.22195 \mathrm{E}-05$ & $6.45 \mathrm{E}-05$ & $8.85542 \mathrm{E}-05$ & 0.00006661 \\
4.5 & $4.05916 \mathrm{E}-05$ & $2.54472 \mathrm{E}-05$ & $2.25 \mathrm{E}-05$ & $3.16869 \mathrm{E}-05$ & $2.29003 \mathrm{E}-05$ \\
5.5 & $1.44099 \mathrm{E}-05$ & $9.61 \mathrm{E}-06$ & $8.62 \mathrm{E}-06$ & 0.000011618 & $8.84 \mathrm{E}-06$ \\
\hline
\end{tabular}

Table 3 Field variable $u$ against distance $x$

\begin{tabular}{|c|c|c|c|c|c|}
\hline$x$ & NCTE & NLS & NGN-II & NGN-III & NMGTE \\
\hline 0.0 & 0 & 0 & 0 & 0 & 0 \\
\hline 0.5 & -0.171014 & -0.113854 & -0.0935007 & -0.157913 & -0.0976409 \\
\hline 1.0 & -0.0540906 & -0.0531456 & -0.0520725 & -0.0536953 & -0.0546321 \\
\hline 1.5 & -0.0137213 & -0.019453 & -0.0209205 & -0.0155647 & -0.020463 \\
\hline 2.0 & -0.00397472 & -0.00767204 & -0.00821823 & -0.00470507 & -0.00728732 \\
\hline 2.5 & -0.00137457 & -0.00378378 & -0.00427237 & -0.0015657 & -0.00402967 \\
\hline 3.0 & -0.00046814 & -0.00199585 & -0.00263436 & -0.00054170 & -0.00265626 \\
\hline 3.5 & -0.00014580 & -0.000991854 & -0.00155047 & -0.00018394 & -0.00154523 \\
\hline 4.0 & $-4.3216 \mathrm{E}-05$ & -0.000461038 & $-8.2600 \mathrm{E}-04$ & $-6.0461 \mathrm{E}-05$ & -0.000777992 \\
\hline 4.5 & $-1.2900 \mathrm{E}-05$ & -0.000208825 & $-4.1300 \mathrm{E}-04$ & $-1.9489 \mathrm{E}-05$ & -0.000364552 \\
\hline 5.5 & $-3.9700 \mathrm{E}-06$ & $-9.58772 \mathrm{E}-05$ & $-2.0500 \mathrm{E}-04$ & $-6.2725 \mathrm{E}-06$ & -0.00017627 \\
\hline
\end{tabular}

Table 4 The non-local stress $\tau_{x x}$ against distance $x$

\begin{tabular}{llllll}
\hline$x$ & CTE & LS & GN-II & GN-III & MGTE \\
\hline 0.0 & -0.408868 & -0.155985 & -0.145709 & -0.333855 & -0.214435 \\
0.5 & -0.184129 & -0.0988131 & -0.0623638 & -0.184029 & -0.0578964 \\
1.0 & -0.0976559 & -0.123225 & -0.126269 & -0.0831271 & -0.13072 \\
1.5 & -0.00191019 & -0.0641543 & -0.0828664 & -0.0327721 & -0.0890927 \\
2.0 & -0.00140958 & -0.022701 & -0.0332923 & -0.0062077 & -0.0300153 \\
2.5 & -0.00109538 & -0.00748039 & -0.0107562 & -0.00129558 & -0.00763243 \\
3.0 & -0.00049598 & -0.00339493 & -0.00475315 & -0.00052566 & -0.00395257 \\
3.5 & -0.00011148 & -0.00189069 & -0.00310482 & -0.00019944 & -0.00314844 \\
4.0 & $-1.8570 \mathrm{E}-05$ & -0.000998984 & -0.00200886 & $-6.6049 \mathrm{E}-05$ & -0.002033 \\
4.5 & $-7.1008 \mathrm{E}-06$ & -0.000476063 & $-1.1300 \mathrm{E}-03$ & $-1.9381 \mathrm{E}-05$ & -0.00105175 \\
5.5 & $-2.2116 \mathrm{E}-06$ & -0.000213832 & -0.000564072 & $-5.4503 \mathrm{E}-06$ & -0.000473434 \\
\hline
\end{tabular}

In all thermoelastic fields, the time changes all the field variables noticeably. In addition, the extracted numerical results justify the significant influence of coefficients' changes on the fields. In contradiction to the coupled and uncoupled theories, results show a limited wave group velocity in both results.

It is evident from the results that $\tau_{0}$ and $K^{*}$ both cause the significant change in the distribution of field quantity. NCTE as coupled theorem and NLS, NGNII, NGNIII and MGTE as non-local generalized theories lead to the same outcomes in the approximation of the half-space surface, due to the dominance of the boundary conditions in that region. On the other hand, within the medium, the solution is substantially different.

The difference between two theories NGN-III and NMGTE is presented in Table 2 and Fig. 6. The magnitude of temperature $\theta$ in NGN-III is shown to be larger than NMGTE. Furthermore, both NLS and NMGTE models indicate the same results. Distributions and especially temperature changes are significantly different in NGN-II from other generalized theories. NGN-III theory in comparison with NGN-II model with low energy dissipation value shows substantially different results. The presence of relaxation time in NLS and NMGTE theories results in a gradual temperature decay. 
Table 5 The strain $e$ against distance $x$

\begin{tabular}{llllll}
\hline$x$ & CTE & LS & GN-II & GN-III & MGTE \\
\hline 0.0 & 0.154293 & 0.0985745 & 0.0811364 & 0.129721 & 0.0864024 \\
0.5 & 0.0353649 & 0.049002 & 0.0493561 & 0.0494183 & 0.0549301 \\
1.0 & 0.0121599 & 0.0198227 & 0.0217495 & 0.0178378 & 0.0218605 \\
1.5 & 0.00561491 & 0.00810607 & 0.0082742 & 0.00663544 & 0.00675295 \\
2.0 & 0.00242289 & 0.00421452 & 0.00380838 & 0.00268781 & 0.00298421 \\
2.5 & 0.000895646 & 0.00263724 & 0.00255834 & 0.0011538 & 0.00245018 \\
3.0 & 0.000238747 & 0.0016596 & 0.00194611 & 0.000499064 & 0.00206128 \\
3.5 & $5.90576 \mathrm{E}-05$ & 0.000973708 & 0.00137519 & 0.000211311 & 0.00145486 \\
4.0 & $2.87545 \mathrm{E}-05$ & 0.000533152 & 0.000871106 & $8.71337 \mathrm{E}-05$ & 0.000875979 \\
4.5 & $1.18972 \mathrm{E}-05$ & 0.000280434 & 0.000505564 & $3.52647 \mathrm{E}-05$ & 0.000469975 \\
5.5 & $4.32000 \mathrm{E}-06$ & 0.000146736 & 0.000280442 & $1.41755 \mathrm{E}-05$ & 0.000241428 \\
\hline
\end{tabular}

The results of NGN-IIII show similar outcomes compared to conventional NCTE. This is completely consistent with the observation of Quintanilla [26] that claimed heat does not dissipate fast through the medium. For viscous mediums, the non-local models NMGTE and NLS follow a similar pattern in the distribution of temperature variation and studied physical variables. Except for the slight difference in the values, both mentioned theories are homologous in behavior. By increasing distance, the results are very similar which is in line with the results of generalized thermoelasticity theorems. We can also notice from Figs. 6,7, 8 that all physical fields in the case of NCTE model are more affected than that of the all other non-local models.

\subsection{The influence of magnetic field}

Figures 10,11, 12 and 13, in the third category, demonstrate the variety of fields studied when $\alpha=0.75$ and $\xi=0.1$ with three assigned values of the magnetic field. The parameter $\varepsilon=\sigma_{0} \mu_{0} H_{x}^{2} /\left(\rho \mathrm{c}_{0}^{2} \omega_{0}\right)$ represents the magnitude of the magnetic field spread over the surface in the direction perpendicular to the $\mathrm{x}$-axis. We will take into account three values of the parameter $\varepsilon$, the first indicates zero magnetic field $(\varepsilon=0)$, and the two other values emphasize the presence of magnetic field $(\varepsilon=1,5)$. The figures show an important influence on the $u, \theta, e$ and the non-local thermal stress $\tau_{x x}$ due to the magnetic field.

In Fig. 10, any rise in the value of parameter $\varepsilon$ induces a decrease in the displacement amounts, which is very noticeable in the curves' peaks. The magnetic field is therefore able to humidify the thermal expansion of the medium. The findings are similar as described in [64]. It can be observed from Fig. 11 that the applied magnetic field has a little influence on the variations of temperature distribution. The temperature changes and slightly increases with the increase in the primary magnetic field. It is the same behavior that many materials and minerals take, and it has been proven in many studies as reported in [71,72].

Figure 12 shows that the rise in parameter $\varepsilon$ increases the value of the non-local stress field $\tau_{x x}$, which is considerable at the start and peak points of the curves. Non-local stress begins with negative values, then increases to their peak and gradually decreases to zero values when the distance $x$ increases. As a result of the fluctuating behavior of the strain $e$, similar to the previous cases, it is noted from Fig. 13 that the strain increases in some intervals by increasing the magnetic field and decreases in other intervals.

\section{Conclusion}

The main aim of this work was to implement a new size-dependent thermoelastic model based on the nonlocal elasticity theory. Moreover, a modified Moore-Gibson-Thompson thermoelastic heat transfer model for heat conduction was introduced. The classical theory of elasticity, the constituent relationships and motion equation have been refined by taking into account the size effect and the commonly adopted models containing the non-local theory proposed by Eringen. The non-local elasticity theories were developed well and used in elastic and thermoelastic solids for the problems of the wave distribution.

In analyzing the propagation of transient waves and vibrations in an infinite thermoelastic materials, the one-dimensional form of the new theory was applied in the presence of a periodic heat sources spread out in the plane region. The techniques of Laplace transform and state space approach were utilized to achieve the closed-form solution for the field variables in the transform domain. In the field of space-time, Zakian's algorithm allows the computational reflections of the studied physical parameters. In some cases, the numerical 
results were displayed graphically and validated. It can be inferred from the discussion and findings that the non-local parameter has a significant influence on the variation of the considered fields. The influence of magnetic field on the system variables was also very important. For the large values of the distance variable, which is the principal feature of non-local thermoelasticity, the fields studied in this new model vanish. This implies that for a certain moment, the thermally disturbed area is attached.

As expected, escalading distance causes the distribution of all physical fields to march to zero, which also indicates the limited group velocity of heat waves. The results discussed in this paper will be useful for the researchers who are working on novel materials and those working on the advancement of thermoelasticity. Finally, our findings can be considered as a theoretical basis for nanoscale structural design, especially for those subjected to varying heat sources.

Acknowledgements Hamid Mohammad-Sedighi is grateful to the Research Council of Shahid Chamran University of Ahvaz for its financial support (Grant No. SCU.EM99.98). VAE acknowledges the support of the Government of the Russian Federation (contract No. 14.Z50.31.0046).

Open Access This article is licensed under a Creative Commons Attribution 4.0 International License, which permits use, sharing, adaptation, distribution and reproduction in any medium or format, as long as you give appropriate credit to the original author(s) and the source, provide a link to the Creative Commons licence, and indicate if changes were made. The images or other third party material in this article are included in the article's Creative Commons licence, unless indicated otherwise in a credit line to the material. If material is not included in the article's Creative Commons licence and your intended use is not permitted by statutory regulation or exceeds the permitted use, you will need to obtain permission directly from the copyright holder. To view a copy of this licence, visit http://creativecommons.org/licenses/by/4.0/.

Funding Open access funding provided by Universitã degli Studi di Cagliari within the CRUI-CARE Agreement.

\section{Declarations}

Funding This study was partially funded by of Research Council of Shahid Chamran University of Ahvaz for its financial support (Grant No. SCU.EM99.98)

Conflict of interest Ahmed E. Abouelregal declares that he has no conflict of interest. Hamid Mohammad-Sedighi declares that he has no conflict of interest. A. H. Shirazi declares that he has no conflict of interest. Mohammad Malikan declares that he has no conflict of interest. Victor A. Eremeyev declares that he has no conflict of interest.

Ethical approval This article does not contain any studies with human participants or animals performed by any of the authors.

\section{References}

1. Biot, M.A.: Thermoelasticity and irreversible thermodynamics. J. Appl. Phys. 27(3), 240-253 (1956)

2. Lord, H.W., Shulman, Y.: A generalized dynamical theory of thermoelasticity. J. Mech. Phys. Solids 15(5), 299-309 (1967)

3. Green, A.E., Lindsay, K.A.: Thermoelasticity. J. Elast. 2(1), 1-7 (1972)

4. Chandrasekharaiah, D.S.: Hyperbolic thermoelasticity: a review of recent literature. Appl. Mech. Rev. 51(12), 705-729 (1998)

5. Tzou, D.Y.: Macro-To Micro-Scale Heat Transfer: The Lagging Behavior. Taylor \& Francis, Abingdon (1997)

6. Marin, M., Öchsner, A., Craciun, E.M.: A generalization of the Gurtin's variational principle in thermoelasticity without energy dissipation of dipolar bodies. Contin. Mech. Therm. 32, 1685-1694 (2020)

7. Ignaczak, J., Ostoja-Starzewski, M.: Thermoelasticity with Finite Wave Speeds. Oxford University Press, Oxford (2010)

8. Hetnarski, R.B., Eslami, M.R., Gladwell, G.M.L.: Thermal Stresses: Advanced Theory and Applications, vol. 4. Springer, New York (2009)

9. Abd-alla, A., Giorgio, I., Galantucci, L., Hamdan, A.M., Del Vescovo, D.: Wave reflection at a free interface in an anisotropic pyroelectric medium with nonclassical thermoelasticity. Contin. Mech. Therm. 28(1-2), 67-84 (2016)

10. Green, A.E., Naghdi, P.M.: A re-examination of the basic postulates of thermomechanics. Proc. R. Soc. Lond. A 432, 171-194 (1991)

11. Green, A.E., Naghdi, P.M.: On undamped heat waves in an elastic solid. J. Therm. Stress. 15, 253-264 (1992)

12. Green, A.E., Naghdi, P.M.: Thermoelasticity without energy dissipation. J. Elast. 31, 189-208 (1998)

13. Abouelregal, A.E.: Modified fractional thermoelasticity model with multi-relaxation times of higher order: application to spherical cavity exposed to a harmonic varying heat. Waves Rand Compl. Med. (2019). https://doi.org/10.1080/17455030. 2019.1628320

14. Abouelregal, A.E.: On Green and Naghdi thermoelasticity model without energy dissipation with higher order time differential and phase-lags. J. Appl. Comput. Mech. (2019). https://doi.org/10.22055/JACM.2019.29960.164

15. Abouelregal, A.E.: Two-temperature thermoelastic model without energy dissipation including higher order time-derivatives and two phase-lags. Mater. Res. Express 1, 21 (2019). https://doi.org/10.1088/2053-1591/ab447f

16. Abouelregal, A.E.: A novel model of nonlocal thermoelasticity with time derivatives of higher order. Math. Method Appl. Sci. (2020). https://doi.org/10.1002/mma.6416 
17. Abouelregal, A.E.: Generalized mathematical novel model of thermoelastic diffusion with four phase lags and higher-order time derivative. Eur. Phys. J. Plus 135, 263 (2020)

18. Abouelregal, A.E.: A novel generalized thermoelasticity with higher-order time-derivatives and three-phase lags. Multidiscip. Model Mater. Struct. 16(4), 689-711 (2019)

19. Dreher, M., Quintanilla, R., Racke, R.: Ill-posed problems in thermo-mechanics. Appl. Math. Lett. 22, 1374-1379 (2009)

20. Quintanilla, R.: Moore-Gibson-Thompson thermoelasticity. Math. Mech. Solids 24, 4020-4031 (2019)

21. Kaltenbacher, B., Lasiecka, I., Marchand, R.: Wellposedness and exponential decay rates for the Moore-Gibson-Thompson equation arising in high intensity ultrasound. Control Cybern. 40, 971-988 (2011)

22. Lasiecka, I., Wang, X.: Moore-Gibson-Thompson equation with memory, part II: general decay of energy. J. Diff. Equ. 259, 7610-7635 (2015)

23. Marchand, R., McDevitt, T., Triggiani, R.: An abstract semigroup approach to the third order Moore-Gibson-Thompson partial differential equation arising in high-intensity ultrasound: structural decomposition, spectral analysis, exponential stability. Math. Methods Appl. Sci. 35, 1896-1929 (2012)

24. Pellicer, M., Sola-Morales, J.: Optimal scalar products in the Moore-Gibson-Thompson equation. Evol. Equ. Control 8, 203-220 (2019)

25. Thompson, P.A.: Compressible-Fluid Dynamics. McGraw-Hill, New York (1972)

26. Quintanilla, R.: Moore-Gibson-Thompson thermoelasticity with two temperatures. Appl. Eng. Sci. 1, 100001 (2020)

27. Dell'Oro, F., Lasiecka, I., Pata, V.: The Moore-Gibson-Thompson equation with memory in the critical case. J Differ Equations 261, 4188-4222 (2016)

28. Dell'Oro, F., Pata, V.: On the Moore-Gibson-Thompson equation and its relation to linear viscoelasticity. Appl. Math. Optim. 76, 641-655 (2017)

29. Pellicer, M., Sola-Morales, J.: Optimal scalar products in the Moore-Gibson-Thompson equation. Evol. Equ. Control Theor. 8, 203-220 (2019)

30. Conti, M., Pata, V., Quintanilla, R.: Thermoelasticity of Moore-Gibson-Thompson type with history dependence in the temperature. Asymptot. Anal. 1, 21 (2019). https://doi.org/10.3233/ASY-191576

31. Marin, M., Othman, M.I.A., Seadawy, A.R., Carstea, C.: A domain of influence in the Moore-Gibson-Thompson theory of dipolar bodies. J. Taibah Univ. Sci. 14(1), 653-660 (2020)

32. Marin, M., Öchsner, A., Bhatti, M.M.: Some results in Moore-Gibson-Thompson thermoelasticity of dipolar bodies. ZAMM J. Appl. Math. Mech. (2020). https://doi.org/10.1002/zamm.202000090

33. Bazarra, N., Fernández, J.R., Quintanilla, R.: Analysis of a Moore-Gibson-Thompson thermoelastic problem. J. Comput. Appl. Math. 382, 15 (2020)

34. Abouelregal, A.E., Ahmed, I.E., Nasr, M.E., Khalil, K.M., Zakria, A., Mohammed, F.A.: Thermoelastic processes by a continuous heat source line in an infinite solid via Moore-Gibson-Thompson thermoelasticity. Materials 13(19), 4463 (2020)

35. Malikan, M., Wiczenbach, T., Eremeyev, V.A.: On thermal stability of piezo-flexomagnetic microbeams considering different temperature distributions. Contin. Mech. Thermodyn. (2021). https://doi.org/10.1007/s00161-021-00971-y

36. Reiher, J.C., Giorgio, I., Bertram, A.: Finite-element analysis of polyhedra under point and line forces in second-strain gradient elasticity. J. Eng. Mech. 143, 04016112-1-13 (2017)

37. Sedighi, H.M.: Divergence and flutter instability of magneto-thermo-elastic C-BN hetero-nanotubes conveying fluid. Acta. Mech. Sin. 36, 381-396 (2020)

38. Malikan, M.: Electro-mechanical shear buckling of piezoelectric nanoplate using modified couple stress theory based on simplified first order shear deformation theory. Appl. Math. Model. 48, 196-207 (2017)

39. Eringen, A.C.: Nonlocal polar elastic continua. Int. J. Eng. Sci. 10, 1-16 (1972)

40. Sedighi, H.M., Daneshmand, F., Abadyan, M.: Modeling the effects of material properties on the pull-in instability of nonlocal functionally graded nano-actuators. Z. Angew Math. Mech. 96, 385-400 (2016)

41. Sedighi, H.M.: The influence of small scale on the pull-in behavior of nonlocal nanobridges considering surface effect, Casimir and van der Waals attraction. Int. J. Appl. Mech. 6(3), 1450030 (2014)

42. Malikan, M., Eremeyev, V.A., Sedighi, H.M.: Buckling analysis of a non-concentric double-walled carbon nanotube. Acta Mech. 231, 5007-5020 (2020). https://doi.org/10.1007/s00707-020-02784-7

43. Malikan, M., Uglov, N.S., Eremeyev, V.A.: On instabilities and post-buckling of piezomagnetic and flexomagnetic nanostructures. Int. J. Eng. Sci. 157, 103395 (2020)

44. Inan, E., Eringen, A.C.: Nonlocal theory of wave propagation in thermoelastic plates. Int. J. Eng. Sci. 29, 831-843 (1991)

45. Wang, J., Dhaliwal, R.S.: Uniqueness in generalized nonlocal thermoelasticity. J. Therm. Stress. 16, $71-77$ (1993)

46. Abouelregal, A.E., Mohammed, W.: Effects of nonlocal thermoelasticity on nanoscale beams based on couple stress theory. Math. Method Appl. Sci. (2020). https://doi.org/10.1002/mma.6764

47. Abouelregal, A.E., Marin, M.: The response of nanobeams with temperature-dependent properties using state-space method via modified couple stress theory. Symmetry 12, 1276 (2020)

48. Koutsoumaris, C., Eptaimeros, K.G., Tsamasphyros, G.J.: A different approach to Eringen's nonlocal integral stress model with applications for beams. Int. J. Solids Struct. 112, 222-238 (2017)

49. Liew, K.M., Zhang, Y., Zhang, L.W.: Nonlocal elasticity theory for grapheme modeling and simulation: prospects and challenges. J. Model Mech. Mater. (2017). https://doi.org/10.1515/jmmm-2016-0159

50. Rajneesh, K., Aseem, M., Rekha, R.: Transient analysis of nonolocal microstretch thermoelastic thick circular plate with phase lags. Mediterr. J. Model. Simul. 9, 025-042 (2018)

51. Javili, A., Morasata, R., Oterkus, E., Oterkus, S.: Peridynamics review. Math. Mech. Solids 24(11), 3714-3739 (2019)

52. Dell'Isola, F., Andreaus, U., Placidi, L.: At the origins and in the vanguard of peridynamics, non-local and higher-gradient continuum mechanics: an underestimated and still topical contribution of Gabrio Piola. Math. Mech. Solids 20(8), 887-928 (2015)

53. Abouelregal, A.E., Mohamed, B.O.: Fractional order thermoelasticity for a functionally graded thermoelastic nanobeam induced by a sinusoidal pulse heating. J. Comput. Theor. Nanos 15, 1233-1242 (2018) 
54. Kostyrko, S., Grekov, M., Altenbach, H.: Stress concentration analysis of nanosized thin-film coating with rough interface. Contin. Mech. Therm. 31, 1863-1871 (2019)

55. Elghamdi, N.A.: The vibration of nano-beam subjected to thermal shock and moving heat source with constant speed. J. Nano Res. 61, 136-150 (2020)

56. Eltaher, M.A., Kabeel, A.M., Almitani, K.H., Abdraboh, A.M.: Static bending and buckling of perforated nonlocal sizedependent nanobeam. Microsyst. Technol. 24, 4881-4893 (2018)

57. Abouelregal, A.E.: Rotating magneto-thermoelastic rod with finite length due to moving heat sources via Eringen's nonlocal model. J. Comput. Appl. Mech. 50(1), 118-126 (2019)

58. Abd-alla, A., Alshaikh, F., Del Vescovo, D., Spagnuolo, M.: Plane waves and eigenfrequency study in a transversely isotropic magneto-thermoelastic medium under the effect of a constant angular velocity. J. Therm. Stress. 40(9), 1079-1092 (2017)

59. Zakian, V.: Numerical inversions of Laplace transforms. Electron. Lett. 327, 120-121 (1969)

60. Zakian, V.: Properties of IMN approximants. In: Graves-Morris, P.R. (ed.) Pade Approximants and Their Applications. Academic Press, London (1973)

61. Halsted, D.J., Brown, D.E.: Zakian's technique for inverting Laplace transform. Chem. Eng. J. 3, 312-313 (1972)

62. Wang, H., Dong, K., Men, F., Yan, Y.J., Wang, X.: Influences of longitudinal magnetic field on wave propagation in carbon nanotubes embedded in elastic matrix. Appl. Math. Model. 34, 878-889 (2010)

63. Mallik, S.H., Kanoria, M.: Generalized thermoelastic functionally graded solid with a periodically varying heat source. Int. J. Solids Struct. 44, 7633-7645 (2007)

64. Das, N.C., Lahiri, A., Sarkar, S.: Eigenvalue value approach three dimensional coupled thermoelasticity in a rotating transversely isotropic medium. Tamsui Oxford J. Math. Sci. 25, 237-257 (2009)

65. Bachher, M., Sarkar, N., Lahiri, A.: Generalized thermoelastic infinite medium with voids subjected to a instantaneous heat sources with fractional derivative heat transfer. Int. J. Mech. Sci. 89, 84-91 (2012)

66. Mondal, S., Sur, A., Kanoria, M.: Magneto-thermoelastic interaction in a reinforced medium with cylindrical cavity in the context of Caputo-Fabrizio heat transport law. Acta Mech. 1, 21 (2019). https://doi.org/10.1007/s00707-019-02498-5

67. Sarkar, N.: Thermoelastic responses of a finite rod due to nonlocal heat conduction. Acta Mech. 231, 947-955 (2020)

68. Roychoudhuri, S.K., Dutta, P.S.: Thermoelastic interaction without energy dissipation in an infinite solid with distributed periodically varying heat sources. Int. J. Solids Struct. 42, 4192-4203 (2005)

69. Bachher, M., Sarkar, N.: Nonlocal theory of thermoelastic materials with voids and fractional derivative heat transfer. Wave Rand. Complex (2018). https://doi.org/10.1080/17455030.2018.1457230

70. Wang, Q., Liew, K.M.: Application of nonlocal continuum mechanics to static analysis of micro- and nano-structures. Phys. Lett. A 363(3), 236-242 (2007)

71. He, T., Cao, L.: A problem of generalized magneto-thermoelastic thin slim strip subjected to a moving heat source. Math. Comput. Model. 49, 1710-1720 (2009)

72. Tian, X.G., Shen, Y.P.: Study on generalized magneto-thermoelastic problems by FEM in time domain. Acta Mech. Sin. 21, 380-387 (2005)

Publisher's Note Springer Nature remains neutral with regard to jurisdictional claims in published maps and institutional affiliations. 